\title{
Detection of extended molecular gas in the disk of the LSB galaxy Malin 2
}

\author{
M. Das ${ }^{1,2}$, F. Boone ${ }^{3}$, and F. Viallefond ${ }^{3}$ \\ 1 Raman Research Institute, Sadashivanagar, Bangalore, 560080, India \\ e-mail: chandaniket@gmail.com \\ 2 Birla Institute of Technology and Science - Pilani, Hyderabad Campus, Jawahar Nagar, Shameerpet Mandal, Hyderabad, 500078, \\ India \\ 3 Observatoire de Paris, LERMA, 61 avenue de l'Observatoire, 75014 Paris, France \\ e-mail: frederic.boone@obspm.fr; fviallef@maat.obspm.fr
}

Received 2 December 2009 / Accepted 4 June 2010

ABSTRACT

\begin{abstract}
Aims. Our goal is to see if there is molecular gas extending throughout the optical low surface brightness disk of the galaxy Malin 2. Methods. We used the heterodyne receiver array (HERA) mounted on the IRAM 30 m telecope to make deep observations at the frequency of the $\mathrm{CO}(2-1)$ line at nine different positions of Malin 2. With a total observing time of $11 \mathrm{~h}$ at a velocity resolution of $11 \mathrm{~km} \mathrm{~s}^{-1}$ we achieve a sensitivity level of $1 \mathrm{mK}$.

Results. We detect $\mathrm{CO}(2-1)$ line emission from Malin 2. The line is detected in four of the nine HERA beams; a fifth beam shows a marginal detection. These results not only confirm that there is molecular gas in the disk of Malin 2, but they also show that it is spread throughout the inner $34 \mathrm{kpc}$ radius as sampled by the observations of the galaxy disk. The mean molecular gas surface density in the disk is $1.1 \pm 0.2 M_{\odot} \mathrm{pc}^{-2}$, and the molecular gas mass lies between the limits $4.9 \times 10^{8}$ to $8.3 \times 10^{8} M_{\odot}$. The observed velocity dispersion of the molecular gas is higher $\left(\sim 13 \mathrm{~km} \mathrm{~s}^{-1}\right)$ than in star-forming galactic disks. This could explain the disk stability and its low star-formation activity.
\end{abstract}

Key words. galaxies: spiral - galaxies: kinematics and dynamics - galaxies: ISM - ISM: molecules - radio lines: ISM galaxies: individual: Malin 2

\section{Introduction}

Low surface brightness (LSB) galaxies are the most unevolved class of galaxies in our nearby Universe (Impey \& Bothun 1997). They are optically dim with diffuse stellar disks (Auld et al. 2006), massive HI gas disks (O'Neil et al. 2004; Matthews et al. 2001) but have low star-formation rates compared to regular spiral galaxies (McGaugh 1994). They are halo-dominated galaxies (de Blok \& McGaugh 1996; Kuzio de Naray et al. 2008; Coccato et al. 2008); this may account for the weak spiral arms and small bar perturbations observed in these galaxies (Mihos et al. 1997; Mayer \& Wadsley 2004). Although the most commonly observed LSB galaxies are the dwarf LSB galaxies (Sabatini et al. 2003), a significant fraction of LSB galaxies are large spirals with prominent bulges (Beijersbergen et al. 1999). These giant LSB (GLSB) galaxies have extended LSB disks that are poor in star formation and dust (Rahman et al. 2007; Hinz et al. 2007). The bulge-dominated GLSB galaxies often show AGN activity (Schombert 1998; Das et al. 2009).

Even though the optical properties of LSB galaxies have been investigated in great depth, not much is known about their molecular gas content. This is important as a knowledge of the cold gas distribution in LSB galaxies will help us understand star-formation processes in these galaxies. Surveys of LSB galaxies show that they have fairly massive HI disks that may be more than twice the size of the optical disk (de Blok et al. 1996; Pickering et al. 1997; Das et al. 2007). In this paper we examine the molecular gas distribution in a GLSB galaxy and see how it relates to the overall star formation in its disk. Molecular gas has been detected in only a handful of LSB galaxies (O'Neil et al. 2000; Matthews \& Gao 2001; O'Neil et al. 2003; Matthews et al. 2005; Das et al. 2006). In most cases the galaxies were large spirals with extended optically dim disks. The low detection rate of molecular emission from LSB galaxies is probably due to several factors related to the poor star-formation rate in these galaxies (e.g. de Blok \& van der Hulst 1998); factors such as the lower dust content, lower metallicity and the lower surface density of cold, neutral gas in these galaxies. All of these properties lead to a slower rate of gas cooling and molecule formation. For the few galaxies where molecular gas has been detected, not much is known about the gas extent and distribution. This information is important if we want to understand star formation and disk evolution in LSB galaxies.

To investigate the molecular gas and star formation in GLSB galaxies we studied the $\mathrm{CO}$ distribution in a galaxy where molecular gas has been detected, F568-6 or Malin 2, as it is widely known (Das et al. 2006). It is a nearly face-on GLSB galaxy at a distance of $201 \mathrm{Mpc}$. It has a prominent bulge and a very extended LSB disk. Its parameters are summarised in Table 1. There are several localized star-forming regions distributed over its inner disk. Its metalicity is one third of the solar metalicity in value, which is relatively high for an LSB galaxy (McGaugh 1994). The CO observations of Malin 2 were conducted using the HERA instrument mounted on the $30 \mathrm{~m}$ IRAM telescope. Our main aim was to examine the molecular gas distribution; determine its extent, total gas mass and surface density. 
Table 1. Galaxy parameters - Malin 2.

\begin{tabular}{lcc}
\hline \hline Parameter & Value & Reference \\
\hline Other names & F568-6 & NED \\
Distance & $201 \mathrm{Mpc}$ & NED \\
Heliocentric velocity & $13830 \mathrm{~km} \mathrm{~s}^{-1}$ & NED \\
Position (epoch 2000) & $10^{\mathrm{h}} 39^{\mathrm{m}} 52^{\mathrm{s}} .5,+20^{\circ} 50^{\prime} 49^{\prime \prime}$ & $2 \mathrm{MASS}$ \\
Size $\left(D_{25}^{\prime}\right)$ & 1.67 & (a) \\
Position angle & $70^{\circ}$ & NED \\
Inclination & $38^{\circ}$ & (a) \\
HI Linewidth $\left(W_{20, \text { corr }}\right)$ & $637 \mathrm{~km} \mathrm{~s}^{-1}$ & (b), (c) \\
HI Mass & $4.2 \times 10^{10} M_{\odot}$ & (b) \\
Dynamical mass & $2.5 \times 10^{12} M_{\odot}$ & $(\mathrm{b})$ \\
$(R<107$ kpc) & & \\
Disk central brightness & $22.1 \mathrm{mag} \mathrm{arcsec}^{-2}$ & (b), (d) \\
Disk scale length & $18.8 \mathrm{kpc}$ & (b) \\
Total $R$ band magnitude & $-23.6 \mathrm{mag}^{\circ}$ & (b) \\
\hline
\end{tabular}

Notes. (a) Matthews et al. (2001). (b) Pickering et al. (1997). (c) Corrected for inclination. (d) Not corrected for extinction.

\section{Observations}

During 2007 March we observed the $\mathrm{CO}(2-1)$ line in Malin 2 with the HERA beam array (Schuster et al. 2004) on the IRAM $30 \mathrm{~m}$ telescope at a fequency of $220.372 \mathrm{GHz}$. We specifically used this array because it has a wide field of view and good sensitivity. HERA is made of nine receivers in a $3 \times 3$ array spaced by $24^{\prime \prime}$ on the sky. The backend used was the Wideband Line Multiple Autocorrelator (WILMA). The total bandwidth was $930 \mathrm{~Hz}$; it was made up of 465 channels of $2 \mathrm{MHz}$ each. The typical system temperatures $T_{\text {sys }}$ were in the range $200-250 \mathrm{~K}$ for eight receivers; one receiver had a systematically higher $T_{\text {sys }}$ in the range $350-450 \mathrm{~K}$. The mean FWHM of each of the nine beams is $11.7^{\prime \prime}$. As our main goal was to detect the CO emission line from the disk of Malin 2, we kept the array fixed on the sky in the standard pointed mode with the central beam pointed to the galactic nucleus; the field was tracked to get deep integrations at fixed positions in the disk. This enabled us to achieve good sensitivity, but at the cost of undersampling the source. We obtained deep observations at nine positions of the galaxy with a total on source-integration time of $11 \mathrm{~h}$ (Fig. 1). The observations were frequency switched. The intrinsic velocity resolution was $2.7 \mathrm{~km} \mathrm{~s}^{-1}$; the data were then smoothed using a Hanning squared function.

We reduced the data with the CLASS software of the GILDAS package ${ }^{1}$ by fitting a first order baseline to all spectra within a window going from -400 to $+400 \mathrm{~km} \mathrm{~s}^{-1}$ about the galaxys' systemic velocity of $13830 \mathrm{~km} \mathrm{~s}^{-1}$. This window was the same for all nine spectra. The noise level is not the same for all receivers; the lowest is $0.7 \mathrm{mK}$ and the highest is $1.6 \mathrm{mK}$ in the $10.9 \mathrm{~km} \mathrm{~s}^{-1}$ channels. The noise is also not uniform across the band. Hence we computed the baseline and the noise in the $-400+400$ window using emission free channels only. All the details are given in Table 2 . The conversion factor from $\mathrm{K}$ to $\mathrm{Jy}$ is $8.6 \mathrm{Jy} \mathrm{K} \mathrm{K}^{-1}$. To convert from the antenna temperature scale $T_{\mathrm{A}}^{*}$ to the main beam temperature $T_{\mathrm{mb}}$ we multiplied $T_{\mathrm{A}}^{*}$ by the factor $F_{\text {eff }} / B_{\text {eff }}=1.67$, where $F_{\text {eff }}$ is the forward-beam efficiency and $B_{\text {eff }}$ is the main-beam efficiency.

\footnotetext{
${ }^{1}$ http://www.iram.fr/IRAMFR/GILDAS
}

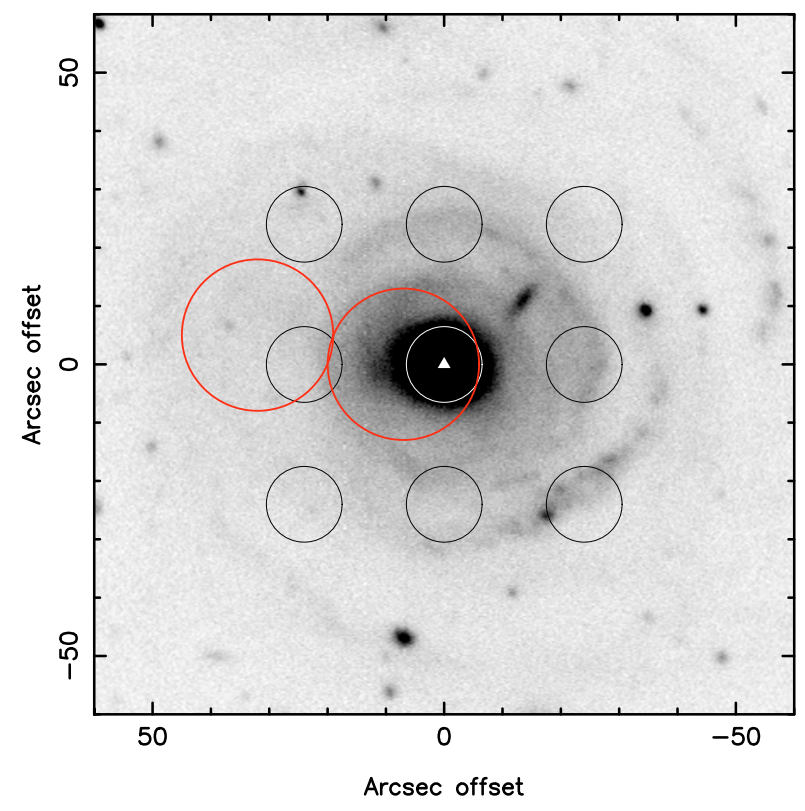

Fig. 1. $R$ band image of the galaxy Malin 2 with the HERA footprint overlaid as nine black circles. The red circles correspond to the previous IRAM observations at $115 \mathrm{GHz}$ (Das et al. 2006). Each circle has a diameter equal to the FWHM of the corresponding beam.

\section{Results}

We have detected $\mathrm{CO}(2-1)$ emission from several positions in the disk as well as from the center of Malin 2. In the paragraphs below we present our results and discuss their implications.

\section{1. $\mathrm{CO}(2-1)$ detections}

Figure 2 shows the $\mathrm{CO}(2-1)$ emission spectra observed from nine locations across Malin 2. The offsets from the galaxy center are indicated in each box. $\mathrm{CO}(2-1)$ emission was detected from four out of nine positions at line intensities above $3 \sigma$. The line at $(-24,24)$ is a a hint of emission rather than a sure detection. At $(0,0)$ the line is broad but is a $3 \sigma$ detection. We estimated the line parameters (flux, width, central velocity) by fitting a Gaussian to each spectrum. We determined the noise level for each of the nine positions. The results are listed in Table 2 and the best-fit Gaussians are overlaid on the spectra in Fig. 2.

\subsection{Comparison with previous $\mathrm{CO}$ observations}

Molecular gas has been detected earlier in this galaxy by Das et al. (2006) with the IRAM 30 m telescope and the BIMA array. However, due to a correlator setup problem the BIMA map was incomplete, it was covering a velocity range corresponding to $\mathrm{CO}(1-0)$ line emission from the east side of the galaxy only. In addition, the $30 \mathrm{~m}$ telescope was pointed toward two directions only at $7^{\prime \prime}$ and $\sim 35^{\prime \prime}$ east of the nucleus (see Fig. 1). Although the source was observed simultaneously at $115 \mathrm{GHz}$ and $230 \mathrm{GHz}$ with the $30 \mathrm{~m}$ telescope, only $\mathrm{CO}(1-0)$ emission was detected; the $\mathrm{CO}(2-1)$ line was not detected from either position. Our present observations cover the $\mathrm{CO}$ emission over a wider velocity and spatial extent than these previous observations and hence give a better idea of the distribution of molecular gas in the inner disk of Malin 2. These observations are also the first detections of the $\mathrm{CO}(2-1)$ line in Malin 2. Our detection lies below the detection limit of the older observations of Das et al. (2006). 
M. Das et al.: Detection of extended molecular gas in the disk of the LSB galaxy Malin 2

Table 2. $\mathrm{CO}(2-1)$ fluxes, kinematical parameters, molecular, HI, and total gas surface densities.

\begin{tabular}{|c|c|c|c|c|c|c|c|c|c|}
\hline \multicolumn{2}{|c|}{$\begin{array}{c}\text { Offset } \\
(\operatorname{arcsec}) \\
a\end{array}$} & $\begin{array}{c}\text { rms noise } \\
(\mathrm{mK}) \\
b\end{array}$ & $\begin{array}{c}\Delta I_{\mathrm{CO}} \\
\left(\mathrm{K} \mathrm{km} \mathrm{s}^{-1}\right) \\
c\end{array}$ & $\begin{array}{c}\Delta V_{F W H M} \\
\left(\mathrm{~km} \mathrm{~s}^{-1}\right) \\
d\end{array}$ & $\begin{array}{c}\langle V\rangle \\
\left(\mathrm{km} \mathrm{s}^{-1}\right) \\
e\end{array}$ & $\begin{array}{c}\Sigma_{\mathrm{mol}} \\
\left(M_{\odot} \mathrm{pc}^{-2}\right) \\
f\end{array}$ & $\begin{array}{c}\Sigma_{\mathrm{HI}} \\
\left(M_{\odot} \mathrm{pc}^{-2}\right) \\
g\end{array}$ & $\begin{array}{c}\sum_{\mathrm{gas}} \\
\left(M_{\odot} \mathrm{pc}^{-2}\right) \\
h\end{array}$ & $\begin{array}{c}\frac{\Sigma_{\text {gas }}}{\sum_{\text {crit }}} \\
i\end{array}$ \\
\hline+24 & +24 & 0.7 & $<0.12$ & 0.39 & . & $<0.4$ & 2.1 & $<3.0$ & \\
\hline+24 & +00 & 0.9 & $0.37 \pm 0.07$ & $82 \pm 20$ & $91 \pm 7$ & $1.2 \pm 0.2$ & 2.5 & 4.8 & 0.7 \\
\hline+24 & -24 & 0.8 & $<0.13$ & 0.45 & $\ldots$ & $<0.5$ & 1.8 & $<2.8$ & $\ldots$ \\
\hline+00 & +24 & 0.9 & $0.28 \pm 0.05$ & $46 \pm 10$ & $14 \pm 4$ & $0.9 \pm 0.2$ & 2.7 & 4.6 & 0.7 \\
\hline+00 & +00 & 0.8 & $0.45 \pm 0.11$ & $243 \pm 76$ & $77 \pm 34$ & $1.5 \pm 0.4$ & 0.9 & 3.4 & \\
\hline+00 & -24 & 0.9 & $<0.15$ & 0.50 & $\ldots$ & $<0.5$ & 2.2 & $<3.3$ & $\ldots$ \\
\hline-24 & +24 & 1.6 & $\sim 0.35 \pm 0.13$ & $79 \pm 41$ & $-102 \pm 14$ & $\sim 1.2 \pm 0.4$ & 2.2 & $\sim 4.4$ & $\sim 0.9$ \\
\hline-24 & +00 & 0.9 & $0.20 \pm 0.04$ & $33 \pm 7$ & $-142 \pm 4$ & $0.7 \pm 0.1$ & 4.0 & 5.7 & 0.9 \\
\hline-24 & -24 & 0.7 & $<0.12$ & 0.39 & $\ldots$ & $<0.4$ & 1.8 & $<2.7$ & $\ldots$ \\
\hline
\end{tabular}

Notes. (a) $\Delta \alpha$ and $\Delta \delta$ offsets relative to the position of the nucleus $\alpha, \delta$ (J2000): $10^{\mathrm{h}} 39^{\mathrm{m}} 52^{\mathrm{s}} .5,+20^{\circ} 50^{\prime} 49^{\prime \prime}$. (b) At $10.9 \mathrm{~km} \mathrm{~s}^{-1}$ velocity resolution. (c) $\Delta I_{\mathrm{CO}(1-0)}=\int T_{\mathrm{MB}} \mathrm{d} v$ with $T_{\mathrm{MB}}=1.67 T_{\mathrm{A}}^{*}$. For the non-detections, we take $T_{\mathrm{A}}^{*}=$ noise and assume $\Delta V=100 \mathrm{~km} \mathrm{~s}^{-1}$. (d) Fitted line widths, not deconvolved by the spectral response. (e) Fitted, relative to the systemic velocity $13830 \mathrm{~km} \mathrm{~s}^{-1}$. (f) $\Sigma_{\mathrm{mol}}=2 m_{\mathrm{p}} N\left(\mathrm{H}_{2}\right)$. Values for $X=$ $2.0 \times 10^{20} \mathrm{~cm}^{-2} \mathrm{~K} \mathrm{~km} \mathrm{~s}^{-1}$ (cf. Sect. 3.4). (g) From Fig. 3 of Pickering et al. (1997). (h) $\Sigma_{\text {gas }}=(1+Z)\left(\Sigma_{\mathrm{HI}}+\frac{X}{20 \times 10^{20}} \Sigma_{\mathrm{mol}}\right) \cos (i)$, with $Z=0.45$, $X=2.8 \times 10^{20} \mathrm{~cm}^{-2} \mathrm{~K} \mathrm{~km} \mathrm{~s}^{-1}$ and $i=38^{\circ}$. Values in brackets assume $\Sigma_{\mathrm{mol}} \ll \Sigma_{\mathrm{HI}}$. (i) Using $\Sigma_{\text {crit }}$ from Fig. 10 of Pickering et al. (1997), re-scaled for $\sigma_{\mathrm{gas}}=13 \mathrm{~km} \mathrm{~s}^{-1}$.
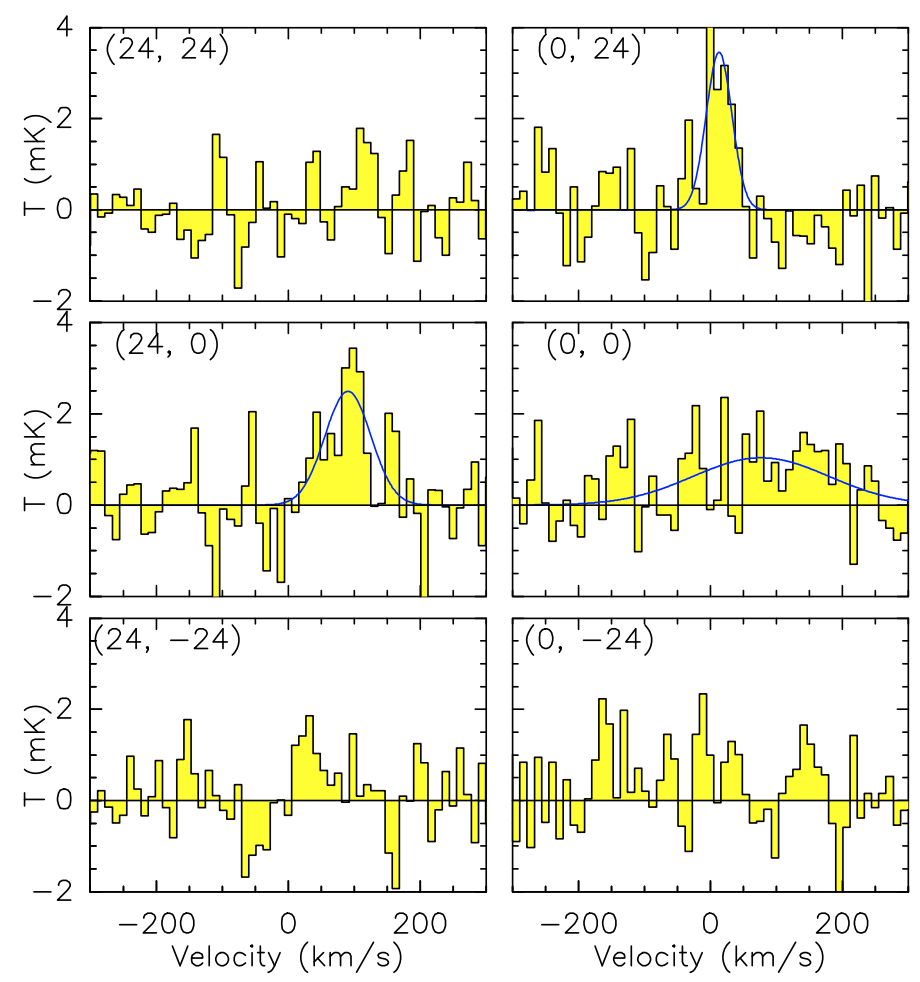

Their $\mathrm{CO}(2-1)$ emission spectrum had a noise (rms) of $2.6 \mathrm{mK}$, whereas our line detections have a peakof 2 to $3 \mathrm{mK}$ (Fig. 2).

Near the galaxy center, where there is overlap with the previous $\mathrm{CO}$ detection, the $\mathrm{CO}$ line velocities are in the same direction and are similar in shape and in width. Close to the center of the galaxy the $\mathrm{CO}(1-0)$ line as detected by Das et al. (2006) is broad $\left(\sim 200 \mathrm{~km} \mathrm{~s}^{-1}\right)$ and asymmetric with the red side slightly more prominent than the blue side. The $\mathrm{CO}(2-1)$ line detected at the center of the galaxy by our new HERA observations is $243 \pm 76 \mathrm{~km} \mathrm{~s}^{-1}$ wide and at a centroid position offset by $77 \pm 34 \mathrm{~km} \mathrm{~s}^{-1}$ relative to the systemic velocity given in Table 1. Investigating the cause of this offset would require interferometric observations. As the beams and pointing directions are different $\left(23^{\prime \prime}\right.$ for the $\mathrm{CO}(1-0)$ line and $11^{\prime \prime}$ for the

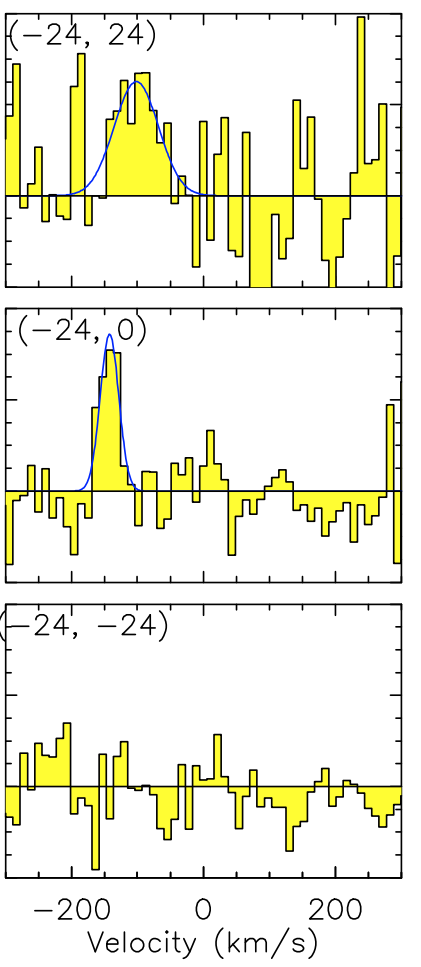

Fig. 2. $\mathrm{CO}(2-1)$ spectra obtained with the nine HERA receivers. The offsets in arcsecond from the galaxy center are marked in the top left hand corner of each figure. $\mathrm{CO}(2-1)$ emission was detected from five locations. The Gaussian fits for these detections are overlaid on the spectra. The $x$-axes are the velocity offsets from the galaxy systemic velocity $\left(v_{\text {hel }}=13830 \mathrm{~km} \mathrm{~s}^{-1}\right)$ and the $y$ axes are the measured antenna temperatures $\left(T_{\mathrm{A}}^{*}\right)$ in $\mathrm{mK}$.

$\mathrm{CO}(2-1)$ line), the fluxes cannot be directly compared. But if the molecular gas were uniformly distributed we would expect a line ratio in temperature scale close to the range of values found by Braine \& Combes (1992) with the same telescope i.e., 0.4 to 1.2. We checked this for the HERA central beam whose pointing direction is the closest to the central pointing of Das et al. (2006). A rough comparison of the brightness in $\mathrm{mK} \mathrm{km} \mathrm{s} \mathrm{k}^{-1}$ gives $I_{\mathrm{CO}(2-1)} / I_{\mathrm{CO}(1-0)}=0.43 / 1.01=0.43$ (where the $\mathrm{CO}(2-$ 1) line intensity from the HERA central pointing is 0.43 and the $\mathrm{CO}(1-0)$ intensity from IRAM is 1.01$)$. This value is at the lower end of the line ratio range for most galaxies, but given the uncertainties (e.g. gas distribution), the value shows that the $\mathrm{CO}(2-1)$ line intensity we measure is consistent with the previous $\mathrm{CO}(1-0)$ detection. 


\subsection{Comparison with $\mathrm{Hl}$ observations}

High-sensitivity HI VLA observations of Malin 2 were presented by Pickering et al. (1997). These reveal the distribution of the HI gas and its kinematics at a spatial resolution of $\sim 19^{\prime \prime}$. The HI disk is lopsided, the HI emission is significantly more prominent and extends farther out on the western side of the nucleus than on the eastern side. The central region is essentialy devoid of HI gas. Although we have only a partial view of the molecular disk owing to our under-sampled data, its properties are similar to HI. Based on the HI velocity field, the centroid velocity of the HI line profiles at the positions $(24,0),(-24,0)$ and $(-24,24)$, respectively $+90,-150$ and $-110 \mathrm{~km} \mathrm{~s}^{-1}$ relative to the systemic velocity, agree excellently with those for the molecular gas (Table 2, Col. e). Hence the HI and molecular gas appear to share a common velocity assymmetry when considering the positions $(24,0)$ and $(-24,0)$.

The higher spatial and spectral resolutions in $\mathrm{CO}$ allow us to better constrain the velocity dispersion of the gaseous component. The CO profile with the narrowest line width is at $(-24,0)$. Given the spectral response, this leads to a deconvolved velocity dispersion of $13 \mathrm{~km} \mathrm{~s}^{-1}$. It is the best constraint that we can get for the velocity dispersion of the gas $\sigma_{\text {gas }}$ in the disk of Malin 2, given that $a$ ) it is obtained in a direction close to the major axis where beam smearing effects due to the rotation of the disk are minimum and $b$ ) we do not observe the blue-shifted wing or secondary velocity component that Pickering et al. discovered and had to blank to determine and analyze the rotation curve. These authors interpreted this secondary component as high-velocity clouds, gas infalling onto the disk southwest of the nucleus. It is best seen $\sim 20^{\prime \prime}$ southwest of the nucleus, where it is blue-shifted by as much as $\sim 100 \mathrm{~km} \mathrm{~s}^{-1}$. Given the correspondance with HII regions in that direction and that there is a relatively moderate $\mathrm{HI}$ column density there, they suggested that this infall induces star formation (Wyder et al. 2009). We do not detect CO emission at $(-24,-24)$, which is only $\sim 6^{\prime \prime}$ further away in the southwest. It could be that the disk component in that region is depeleted not only in HI, but also in molecular gas. Spatially fully sampled CO observations would be required to confirm this hypothesis.

In Table 2, the maximum $\mathrm{CO}(2-1)$ line velocities are reached in the offsets $(24,0)$ and $(-24,0)$, which is consistent with the position angle being close to horizontal $\sim 70^{\circ}$ (2MASS images, Jarrett et al. 2003). We note a velocity asymmetry between the emission at $(24,0)$ and that at $(-24,0)$, which could be due to infalling gas or a lopsided potential. We can estimate the rotational velocity from these lines. Assuming an inclination of $38^{\circ}$ (Matthews et al. 2001) it is on the order $\left(\sim 150 / \sin (i) \mathrm{km} \mathrm{s}^{-1}\right.$, which implies a dynamical mass within the $24^{\prime \prime}$ (i.e. $23 \mathrm{kpc}$ ) radius of $\sim 3 \times 10^{11} M_{\odot}$.

The broad line at the center has a width close to twice the velocities at $(24,0)$ and $(-24,0)$ offsets. This could be because the rotation curve turning point is inside the central beam, i.e., inside a radius of $6^{\prime \prime}(6 \mathrm{kpc})$. This central region, which is essentially devoid of $\mathrm{HI}$, is where the strongest emission in $\mathrm{CO}$ originates; higher angular resolution $\mathrm{CO}$ observations are required to determine the rotation curve in that region. If the curve continued to steadily decrease towards the nucleus, it would point to a model where the gas in the central bulge region is kinematically hot, e.g. because of gas infall to feed the AGN.

\subsection{Molecular gas mass}

We derived the molecular gas surface densities for each line detection and the results are summarised in Table 2.
The column densitites are derived from the line fluxes using $N\left(\mathrm{H}_{2}\right)=X(1 / 0.9) \int T_{\mathrm{mb}}[\mathrm{CO}(2-1)] \mathrm{d} v$; where $I(2-1) / I(1-$ $0)=0.9$ (Braine \& Combes 2000) and the $\mathrm{CO}$ to $\mathrm{H}_{2}$ conversion factor is given by $X=2 \times 10^{20} \mathrm{~cm}^{-2}\left(\mathrm{~K} \mathrm{~km} \mathrm{~s}^{-1}\right)$ for cool virialized molecular clouds (Dickman et al. 1986). The value of $X$ is somewhat uncertain for LSB disks, given their low metalicities and our poor knowledge of the ISM conditions in these galaxies. However, for a first estimate we used this value to determine the surface densities of molecular gas $\Sigma_{\text {mol }}$ in Malin 2 (Col. f, Table 2). Assuming the eight directions observed in the disk form a distribution of surface brightness values which is representative for the disk of Malin 2 up to a radius of $40^{\prime \prime}$, the mean $\mathrm{CO}(2-1)$ brightness is $\sim 0.15 \mathrm{~K} \mathrm{~km} \mathrm{~s}^{-1}$. The central region has a beam-averaged surface density that is three times higher than the mean brightness of the disk. The lower limit for $M_{\mathrm{mol}}$ is $\sim 4.9 \times 10^{8} M_{\odot}$ and is given by the sum of the four detections in Col. f of Table 2 multiplied by the beam solid angle. To determine the upper limit for $M_{\text {mol }}$, we used the noise (see Table 2, Col. a) to derive upper limits for the molecular gas surface densities for the regions where there were no detections including $(-24,24)$, where there is a hint of emission. The upper limit to the total mass of molecular gas within $R<40^{\prime \prime}$ is thus $M_{\text {mol }} \sim 8.3 \times 10^{8} M_{\odot}$. If the molecular disk does not extend beyond $40^{\prime \prime}$, then the molecular gas mass corresponds to $\sim 1.2-2 \%$ of the HI gas mass in the galaxy. This is similar to what is observed in normal spiral galaxies that have molecular gas masses typically a few percent of the total HI mass.

\subsection{Star-formation threshold}

The molecular gas disk in Malin 2 is extended and relatively massive with a significant amount of molecular gas, but its starformation activity is low compared to normal spirals (Wyder et al. 2009). For galactic disks star formation appears to be controled by the onset of gravitational instabilities (Kennicutt 1989). A simple single-fluid Toomre-disk stability model predicts threshold densities in agreement with the observations. This critical density is given by $\Sigma_{\text {crit }}=\alpha \kappa \sigma_{\text {gas }} / 3.36 \mathrm{G}$ where $\kappa$ is the epicyclic frequency and $\sigma_{\text {gas }}$ the velocity dispersion of the gas. From a sample of spiral galaxies Kennicutt found $\alpha \simeq$ 0.69 . In his study, to evaluate $\alpha$, Kennicutt assumed that all the galaxies in his sample have approximately the same velocity dispersion, $\sim 6 \mathrm{~km} \mathrm{~s}^{-1}$, and that it is independent of the radial distance within a galaxy. This is a good approximation given what is known for the galaxies of his sample. Hence the prediction is that to have disk galaxies such as the LSBs immune to star formation, the gas surface density should be below this critical density.

Pickering et al. (1997) determined rotation curves for four GLSBs. These allow them to determine the epicyclic frequency necessary to obtain the radial profiles of $\Sigma_{\text {crit }}$. They show that by assuming that $\alpha=0.69$ is valid for these LSB galaxies and that $\sigma_{\text {gas }}=10 \mathrm{~km} \mathrm{~s}^{-1}$, the critical density is in some regions close or lower than the observed HI surface density, although there is no conspicuious star-formation activity there. This leads them to postulate that $\sigma_{\text {gas }}$ must be higher than the assumed value.

In the specific case of Malin 2 Pickering et al. find that the HI surface density never exceeds the critical density except at the location where $\mathrm{HI}$ is the brightest (this direction is located a half way between the positions $(-24,24)$ and $(-24,0))$. When considering the nine directions listed in Table 2 only $(0,0)$ coincides with an $\mathrm{H} \alpha$ source. Using the present $\mathrm{CO}$ observations we can examine two questions: 1) does the gas surface density 
remain below the critical density when the contribution from the molecular gas component is taken into account as did Kennicutt in his analysis? 2) Is $\sigma_{\text {gas }} \leq 10 \mathrm{~km} \mathrm{~s}^{-1}$ used to determine $\Sigma_{\text {crit }}$ adapted in the case of giant LSBs such as Malin 2? Column $\mathrm{g}$ in Table 2 gives the HI surface brightnesses. Comparing these with the molecular surface densities (Col. f), the molecular gas surface densities appear to amount to typically $1 / 2$ to $1 / 3$ the HI surface densities, with the exception of position $(-24,0)$, where HI largely dominates. Column $\mathrm{h}$ gives the total gas surface density and in Col. $\mathrm{i}$ this quantity relative to the critical density. By using $\alpha=0.69$, for internal consistency, the values in Cols. $\mathrm{h}$ and $\mathrm{i}$ are determined using the same hypothesis as that adopted by Kennicutt for the conversion factor $X$ and the term $Z$, which accounts for the elements heavier than $\mathrm{H}$. For $\sigma_{\text {gas }}$ we adopted the value of $13 \mathrm{~km} \mathrm{~s}^{-1}$ that we measured with good $\mathrm{S} / \mathrm{N}$ at $(-24,0)$ (Sect. 3.3). Better constrained than the $10 \mathrm{~km} \mathrm{~s}^{-1}$ adopted by Pickering et al., this value is still conservative given that it is the lowest velocity dispersion (Col. d) amongst the five directions where CO is detected. Formally, because all the values in Col. i are smaller than unity, it could be concluded that the disk of Malin 2 is sufficiently stable to prevent star formation. However, the directions $(-24,0)$ and $(-24,24)$ have surface densities close to the threshold values, thus preventing us from considering this as a firm result. Also the observed kinematics in western and north-western parts of Malin 2 appears complex because of highvelocity clouds (HVC). It could be that the star-formation activity seen in Malin 2 is more related to the interaction of these HVCs with the disk than to growth of density fluctuations originating from instabilities in the self-graviting rotating disk. A fully sampled image of the Malin 2 and high signal-to-noise to seperate the different velocity components and measure the line widthes are necessary to better understand the properties of Malin 2.

\section{Conclusions}

1. We report the first detection of $\mathrm{CO}(2-1)$ line emission from the disk of a giant LSB galaxy. Our observations reveal extended molecular gas in the disk of the LSB galaxy Malin 2. The radial extent of the molecular disk is at least $34 \mathrm{kpc}$ and its mean line brightness $\sim 0.15 \mathrm{~K} \mathrm{~km} \mathrm{~s}^{-1}$ (on the $T_{\mathrm{mb}}$ temperature scale). With an angular resolution of $11.7^{\prime \prime}$ this is $\sim 2 / 3$ the beam-averaged integrated line intensity in the central region.

2. When we compared the molecular and HI gas masses of Malin 2, we found that the molecular gas fraction is $\sim 1.2-$ $2 \%$ of the $\mathrm{HI}$ gas mass. At radii $\sim 15 \mathrm{kpc}$ in the directions where $\mathrm{CO}$ is detected, the molecular gas fraction is typically $30 \%$ to $50 \%$ of the HI gas surface densities.
3. We estimated the total surface density of the neutral gas and found that it is always below the critical threshold density for gravitational instability. Hence the disk of Malin 2 is overall stable against large scale star-formation.

Acknowledgements. We are grateful to the IRAM staff at Pico Veleta for excellent support at the telescope. IRAM is supported by INSU/CNRS (France), MPG (Germany) and IGN (Spain). The authors would also like to thank Alice Quillen for providing the $R$-band images of Malin 2. This research has made use of the NASA/IPAC Extragalactic Database (NED) which is operated by the Jet Propulsion Laboratory, California Institute of Technology, under contract with the National Aeronautics and Space Administration.

\section{References}

Auld, R., de Blok, W. J. G., Bell, E., \& Davies, J. I. 2006, MNRAS, 366, 1475 Beijersbergen, M., de Blok, W. J. G., \& van der Hulst, J. M. 1999, A\&A, 351, 903

Braine, J., \& Combes, F. 1992, A\&A, 264, 433

Coccato, L., Swaters, R. A., Rubin, V. C., D’Odorico, S., \& McGaugh, S. S. 2008, A\&A, 490, 589

Das, M., O’Neil, K., Vogel, S. N., \& McGaugh, S. 2006, ApJ, 651, 853

Das, M., Kantharia, N., Ramya, S., et al. 2007, MNRAS, 379, 11

Das, M., Reynolds, C. S., Vogel, S. N., McGaugh, S. S., \& Kantharia, N. G. 2009, ApJ, 693, 1300

Dickman, R. L., Snell, R. L., \& Schloerb, F. P. 1986, ApJ, 309, 326

de Blok, W. J. G., McGaugh, S. S., \& van der Hulst, J. M. 1996, MNRAS, 283, 18

de Blok, W. J. G., \& van der Hulst, J. M. 1998, A\&A, 336, 49

Hinz, J. L., Rieke, M. J., Rieke, G. H., et al. 2007, ApJ, 663, 895

Impey, C., \& Bothun, G. 1997, ARA\&A, 35, 267

Jarrett, T. H., Chester, T., Cutri, R., Schneider, S. E., \& Huchra, J. P. 2003, AJ, 125,525

Kennicutt, R. C. 1989, ApJ, 344, 685

Kuzio de Naray, R., McGaugh, S. S., \& de Blok, W. J. G. 2008, ApJ, 676, 920

Matthews, L. D., \& Gao, Y. 2001, ApJ, 549, L191

Matthews, L. D., van Driel, W., \& Monnier-Ragaigne, D. 2001, A\&A, 365, 1

Matthews, L. D., Gao, Y., Uson, J. M., \& Combes, F. 2005, AJ, 129, 1849

Mayer, L., \& Wadsley, J. 2004 MNRAS, 347, 277

McGaugh, S. S. 1994, ApJ, 426, 135

McGaugh, S. S., Bothun, G. D., \& Schombert, J. M. 1995, AJ, 110, 573

Mihos, J. C., McGaugh, S. S., \& de Blok, W. J. G 1997, ApJ, 477L, 79

O’Neil, K., Hofner, P., \& Schinnerer, E. 2000, ApJ, 545, L102

O'Neil, K., Schinnerer, E., \& Hofner, P. 2003, ApJ, 588, 230

O’Neil, K., Bothun, G., van Driel, W., \& Monnier Ragaigne, D. 2004, A\&A, 428,823

Pickering, T. E., Impey, C. D., van Gorkom, J. H., \& Bothun, G. D. 1997, AJ, 114,1858

Rahman, N., Howell, J. H., Helou, G., Mazzarella, J. M., \& Buckalew, B. 2007, ApJ, 663, 908

Schuster, K.-F., Boucher, C., Brunswig, W., et al. 2004, A\&A, 423, 1171

Sabatini, S., Davies, J., Scaramella, R., et al. 2003, MNRAS, 341, 981

Schombert, J. M. 1998, AJ, 116, 1650

Wyder, T. K., Martin, D. C., Barlow T. A., et al. 2009, ApJ, 696, 1834 